\title{
Validez y Confiabilidad del Inventario de Expresión de Ira (DAX) en Conductores de Argentina
}

\author{
Validity and Reliability of the Driver Anger Expression Inventory (DAX) in \\ Argentinean Drivers
}

\author{
Mario A. Trógolo ${ }^{1}$, Pablo E. Flores Kanter ${ }^{2}$ y Leonardo A. Medrano ${ }^{3}$
}

\begin{abstract}
Resumen
El objetivo de este estudio fue examinar las propiedades psicométricas del Driver Expression Inventory (DAX) para su uso en Argentina. Se aplicó la escala a 378 conductores (hombres $=50.3 \%$ ) con edades entre 18 y 77 años de la población general de Córdoba, Argentina. Mediante análisis factorial no restricto se identificaron cinco factores, congruentes con los propuestos en la escala original. Los índices de consistencia interna fueron satisfactorios para todos los factores $(\alpha=.78-.85)$. Asimismo, se correlacionaron las dimensiones del DAX con medidas de ira y de comportamiento en la conducción, encontrándose relaciones significativas y coherentes en términos teóricos, obteniéndose así evidencia concurrente. Finalmente, por medio de análisis de regresión jerárquica se obtuvo evidencia de validez incremental del DAX. En conjunto, los resultados avalan la validez y fiabilidad del DAX para su utilización en el contexto Argentino, aunque se requieren de nuevos estudios que analicen propiedades psicométricas adicionales.
\end{abstract}

Palabras clave: expresión de la ira, conductores, adaptación, Argentina

\begin{abstract}
The purpose of the current study was to examine psychometric properties of the Driver Expression Inventory (DAX) in Argentina. A sample of 378 drivers (males=50.3\%) aged 18 to 77 years old from the general population of Cordoba, Argentina, participated in the study. Unrestricted factor analysis yielded five factors consistent with the original scale. Internal consistency were satisfactory for all DAX subscales $(\alpha=.78-.85)$. Correlations between DAX and measures of driving anger and drivers' behavior provided concurrent validity. Finally, hierarchical regression analyses showed additional and significant contribution of the DAX in predicting driving-related behaviors beyond demographic variables (i.e. age and gender) and driving anger, supporting for incremental validity. Overall, results support the validity and reliability of the DAX in Argentina, although we note that further studies are needed in order to evaluate additional psychometric properties.
\end{abstract}

Keywords: driving anger expression, adaptation, Argentina

\footnotetext{
${ }^{1}$ Licenciado en Psicología. Docente e Investigador. Avenida Colón 870 departamento PB 4 (CP: 5000). Córdoba, Argentina. Tel.: 0054-0351-158061362. Correo: mario.trogolo@ gmail.com

${ }^{2}$ Licenciado en Psicología. Docente e Investigador. CP: 5000. Leonor de Tejeda 1866 (CP: 5000). Córdoba, Argentina. Tel.: 0054-0351-153849201.Correo: ezequielfk@gmail.com

${ }^{3}$ Doctor en Psicología. Secretario de Investigación, Docente y Investigador. Del Cid 321 (CP: 5000). Villa Allende, Argentina. Tel.:0054-0351-153874871. Correo: leomedpsico@gmail.com
} 
Introducción

El desarrollo de los medios de transporte han cambiado sustancialmente la forma en que vivimos pero, al mismo tiempo, también la que morimos. Cada año mueren en el mundo más de un millón doscientas mil personas por accidentes de tránsito y entre veinte y cincuenta millones sufren lesiones de diversa gravedad (OMS, 2013). En Argentina, el panorama parece ser aún más preocupante (Escanés, Agudelo-Botero, \& Cardona, 2014; Fleitas, 2010; Leveau \& Úbeda, 2012). Diversas estimaciones señalan que las lesiones de tránsito constituyen la primera causa externa de muerte en los jóvenes (Ministerio de Salud de la Nación Argentina, 2013). Los registros hospitalarios muestran, asimismo, que las lesiones de tránsito representan el segundo motivo de ingreso al hospital, detrás de los motivos médicos generales (Escalante, Gómez, Cuasnicu, \& Ansaldo, 2013). Finalmente, algunos estudios señalan que el número de víctimas fatales debidas al tránsito ha evidenciado una tendencia creciente a partir del 2000 (Fleitas, 2010), hecho que ha resultado en una disminución de la esperanza de vida (Escanés et al., 2014).

Aunque los siniestros viales representan eventos complejos que involucran la interacción de diferentes elementos (Petit, 2014), existe acuerdo en que el factor humano representa su elemento más definitorio, estimándose que alrededor del $90 \%$ de los accidentes de tránsito responden a factores derivados del conductor (Evans, 1996). Dentro de estos factores, se han identificado las emociones como un elemento relevante (Alonso, Esteban, Catalayud, Alamar, \& Egido, 2006), particularmente la ira, siendo una de las emociones más estudiadas probablemente debido a su frecuencia y a su vinculación con comportamientos agresivos y arriesgados (Dahlen \& Ragan, 2004; Li, Yao, Jiang, \& Li, 2014; Maxwell, Grant, \& Lipkin, 2005; Trógolo, Flores Kanter, Pareja, \& Medrano, 2015) como por sus efectos negativos sobre la tarea de conducción, incluyendo la pérdida de control del vehículo, la disminución de la concentración, desviaciones y accidentes (Deffenbacher, Lynch, Filleti, Dahlen, \& Oetting, 2003; Jeon, Walker, \& Yim, 2014; Sullman, Stephens, \& Yong, 2014).
$\mathrm{Si}$ bien existe relación entre los comportamientos agresivos y arriesgados, es importante mencionar que se trata de constructos distintos (Poó, Taubman-Ben-Ari, Ledesma, \& Díaz Lázaro, 2013; Taubman-Ben-Ari, Mikulincer, \& Gillath, 2004; Trógolo \& Flores, Kanter, 2016). Así, aun cuando los comportamientos en algunos casos pueden ser similares y suponer un riesgo para otros usuarios (e.g., conducir a alta velocidad), la conducción agresiva representa comportamientos motivados por la impaciencia, irritación, hostilidad y/o intención de ahorrar tiempo (Tasca, 2000). En cambio, no todo comportamiento riesgoso implica reacciones de ira o enojo, sino que en muchas ocasiones puede ser la consecuencia de lucirse ante pares, divertirse o experimentar emociones nuevas (Poó, 2014). De esta forma, los comportamientos agresivos al volante pueden llevar a involucrarse en acciones de riesgo, pero no toda conducta arriesgada supone la presencia de comportamientos agresivos.

Además de la ira, para entender su influencia sobre el comportamiento de los conductores se ha señalado la importancia de tener en cuenta la manera en que es expresada (Deffenbacher, Lynch, Oetting, \& Swaim, 2002; HerreroFernández, 2011). La relevancia de esto radica en el hecho de que dos conductores que experimentan el mismo grado de ira pueden expresarla de diferentes formas. Así, mientras que algunos conductores en estado de ira pueden descargar su bronca verbalmente y/o intentar forzar a un costado del camino al otro conductor, otros en ese mismo estado pueden reconocer que no vale la pena implicarse en el asunto. Tales formas de expresión ejercen una modulación de la activación emocional, afectando la experiencia de ira y los comportamientos durante la conducción (cf., Deffenbacher et al., 2002).

Con el objetivo de analizar las distintas formas de expresión de ira al volante, Deffenbacher et al. (2002) crearon el Driver Anger Expression Inventory (DAX), un instrumento de auto-informe de 53 ítems que valora cinco modos de expresión: verbal, física, mediante el vehículo, desplazada y adaptativaconstructiva. La forma verbal comprende la expresión de la ira a través de insultos, gritos y comentarios negativos a otros conductores. Dentro 
de este factor se incluyen, además, algunas conductas no verbales (e.g., mirar fijamente, mover desaprobatoriamente la cabeza) que acompañan a la agresión verbal. La expresión física comprende el uso del propio cuerpo como medio de intimidación y de expresión de la ira. Abarca comportamientos tales como hacer gestos hostiles con las manos hasta agresiones físicas y establecimiento de peleas. La expresión mediante el vehículo comporta acciones que implican el uso del vehículo mismo como medio de expresión. Supone actos como conducir a muy corta distancia del vehículo del conductor que provocó la ira, acelerar o desacelerar repentinamente para fastidiarlo, o levantar las luces con el objeto de encandilar. A diferencia de las tres formas descritas, que suponen una expresión agresiva directa de la ira contra aquello que la ocasionó, la expresión desplazada representa la expresión de la ira hacia algo o alguien que no es el objeto de provocación. De manera específica, la expresión desplazada consistiría fundamentalmente en la expresión agresiva contra los acompañantes a bordo del propio vehículo de un estado de ira que se genera esencialmente en base a las contingencias causadas por otros usuarios de la vía (Wiesenthal, Hennessy, \& Gibson, 2000). Por último, la forma adaptativa-constructiva supone expresar esta emoción de una manera no agresiva, como por ejemplo por medio de comportamientos asertivos, o bien a través de la relajación o la aceptación de la situación que desencadenó la ira.

$\mathrm{Si}$ bien la propuesta original del DAX comprende cinco factores relativos a las diferentes formas de expresión, la expresión desplazada fue finalmente suprimida por su baja consistencia interna. De este modo, la estructura factorial quedó conformada por cuatro factores y 49 ítems. Las tres formas agresivas de expresión de la ira (i.e., verbal, física y mediante el vehículo) mostraron correlaciones positivas moderadas entre sí y negativas o nulas con la forma de expresión adaptativa-constructiva. Adicionalmente, se obtuvieron correlaciones entre las distintas formas de expresión de la ira y el nivel de ira en la conducción, como así también con los comportamientos arriesgados y agresivos en el tráfico, de forma que los distintos modos de expresión agresiva de la ira se relacionaron con un mayor nivel de ira y una implicación en comportamientos agresivos y arriesgados, mientras que la expresión adaptativa-constructiva presentó un patrón de relaciones opuestas con dichas variables. Finalmente, por medio de distintos análisis de regresión jerárquica se verificó la contribución independiente de las escalas del DAX sobre los comportamientos de conducción arriesgados y agresivos, por encima de sexo y la ira. Estos resultados han sido replicados en estudios posteriores por los mismos autores (Deffenbacher, Kemper, \& Richards, 2007; Deffenbacher, Lynch, Deffenbacher, \& Oetting 2001; Deffenbacher, White, \& Lynch, 2004), ratificando la validez y la utilidad de la escala.

El DAX ha sido traducido a diferentes idiomas y objeto de adaptación en varios países, incluyendo Francia (Villeux \& Delhomme, 2010), España (Gras et al., 2015; Herrero-Fernández, 2011), Malasia (Sullman, Stephens, \& Yong, 2015), Rumania (Sârbescu, 2012), Turquía (Esiyok, Yasak, \& Korkusuz, 2007), Nueva Zelanda (Sullman, 2015), Inglaterra (Stephens \& Sullman, 2014) y China (Ge, Qu, Zhang, Zhao, \& Zhang, 2015), fundamentalmente la versión de 49 ítems que excluye el factor de expresión desplazada. Los resultados obtenidos en las diferentes investigaciones han sido inconsistentes. Así, existen estudios que confirman la estructura de cuatro factores (Sulman, 2015, Sullman et al., 2013, 2015), si bien tras realizar numerosas reespecificaciones al modelo inicial sin una justificación teórica clara, e incluso cuando algunos índices de ajuste sugieren lo contrario. $\mathrm{La}$ validez de estos resultados debe ser tomada con reserva, en la medida que los modelos sobremanipulados basados únicamente en criterios estadísticos suelen ser muy inestables y, por lo tanto, difíciles de replicar en otras muestras (Furr, 2011). Otras investigaciones, en cambio, obtuvieron una composición factorial distinta. Puntualmente, Sârbescu (2012) halló que la escala original del DAX no presentó un buen ajuste en los datos en una muestra de conductores rumanos. Tras inspeccionar los ítems y eliminar aquellos con contenidos redundantes, se obtuvo una nueva versión de 30 ítems y tres factores: expresión adaptativa-constructiva, expresión mediante el vehículo, y expresión física-verbal, que agrupa ítems correspondientes a las escalas originales de 
expresión física y expresión verbal de la ira. De manera similar, en la adaptación francesa Villieux y Delhomme (2010) suprimieron varios ítems redundantes reduciendo la escala a 11 ítems y a través de análisis factoriales exploratorio y confirmatorio evidenciaron tres formas de expresión de ira en conductores franceses: expresión adaptativa-constructiva, expresión mediante el vehículo y expresión no verbal. Finalmente, en la versión española (HerreroFernández, 2011) se confirmó la estructura factorial de cinco maneras de expresión, equivalentes a las originales, incluyendo el factor expresión desplazada de la ira que se mantuvo al encontrarse una adecuada consistencia interna.

Por otra parte, se han llevado a cabo estudios que analizan diferencias en la expresión de la ira en base a diferentes variables como el sexo, la edad, y variables asociadas a la conducción, específicamente los años de experiencia y la frecuencia de conducción. En el caso de la edad, los resultados han sido homogéneos, identificándose a los conductores jóvenes como los más propensos a expresar la ira agresivamente -ya sea verbalmente, físicamente o mediante el vehículo-, mientras que los conductores de más edad tienden a utilizar formas de expresión más adaptativa-constructiva (Deffenbacher et al., 2007; Jovanović, Lipovac, Stanojević, \& Stanojević, 2011; Sârbescu, 2012; Stephens \& Sullman, 2014; Sullman, 2015; Sullman et al., 2015; Villeux \& Delhomme, 2010). En cuanto al sexo, los resultados son más contradictorios, encontrándose mayor expresión agresiva de la ira en los hombres, principalmente la expresión física y mediante el uso del vehículo, y mayor expresión adaptativa-constructiva en las mujeres (Deffenbacher et al., 2004; Gras et al., 2015; Esiyok et al., 2007 Sullman et al., 2015). Sin embargo, otros estudios no hallaron diferencias (Deffenbacher et al., 2007; Ge et al., 2015; Herrero-Fernández, 2011; Villeux \& Delhomme, 2010), o bien encontraron que las mujeres expresan la ira en forma más agresiva, particularmente de manera verbal (Dahlen \& Ragan, 2004).

Por su parte, los estudios que analizan el efecto de la experiencia en la conducción (años de conducción) sobre la expresión de ira parecen indicar que la experiencia de conducción se encuentra asociada a una disminución de las formas agresivas de expresión de la ira, y un aumento en la expresión adaptativa-constructiva (Jovanović et al., 2011; 2011; Stephens \& Sullman, 2014; Sullman et al., 2013, 2015). No obstante, dado que, por un lado, el patrón de correlaciones encontrado es similar a las relaciones entre la edad y la expresión de la ira, y que, por el otro, la edad y los años de experiencia evidencian correlaciones positivas elevadas en todos los casos - sugiriendo, por lo tanto, un solapamiento -, no resulta claro hasta qué punto las diferencias encontradas en la expresión de la ira podrían ser atribuibles a la edad o los años de experiencia.

Por último, la evidencia en torno al papel de la frecuencia de conducción muestra resultados divergentes. Así, algunos estudios señalan que los conductores que manejan con mayor frecuencia tienden a expresar la ira de manera agresiva (Ge et al., 2015; Sullman, 2015; Sullman et al., 2013, 2015), concluyendo que tal situación se originaría en la exposición diferencial a eventos frustrantes en el contexto del tráfico, que daría lugar a una mayor probabilidad de experimentar ira $y$ reaccionar agresivamente. En cambio, Deffenbacher et al. $(2007,2010)$ y Villeux y Delhomme (2010) no hallaron relaciones entre la frecuencia de manejo y la expresión de la ira.

Cabe destacar que la mayoría de las investigaciones sobre el DAX se han realizado con poblaciones universitarias, especialmente estudiantes de psicología (e.g. Deffenbacher et al., 2001, 2002, 2004, 2007; Esiyok et al., 2007; Gras et al., 2015; Herrero-Fernández, 2011; Villieux \& Delhomme, 2010). En comparación, pocos estudios han analizado las propiedades psicométricas de la escala en conductores de la población general (e.g. Ge et al., 2015; Stephens \& Sullman, 2014; Sullman, 2015), siendo la evidencia en estos casos mixta. Por lo tanto, se requieren nuevas investigaciones que analicen las características psicométricas del DAX en poblaciones más generales.

El presente trabajo tuvo por objetivo analizar la validez y confiabilidad de la escala DAX en conductores de la población general de la ciudad de Córdoba, Argentina. Aun cuando a nivel internacional existen varios estudios sobre la expresión de ira en conductores, la falta de 
uniformidad cultural entre los países (Casullo, 2009) y las diferencias culturales en la expresión de la ira en general (Moheb \& Ram, 2010) y en el tránsito en particular (Sârbescu, Stanojević, \& Jovanović, 2014) justifican la necesidad de llevar a cabo estudios locales. Al mismo tiempo, no hemos encontrado estudios que analicen la expresión de la ira al volante en Argentina, lo que podría deberse en parte a la carencia de instrumentos para su evaluación. Por consiguiente, el presente trabajo consistió en analizar las cualidades psicométricas del DAX. Específicamente, se exploró la estructura interna y la consistencia de la escala. A continuación, se analizaron diferencias en las puntuaciones del DAX en función del sexo, la edad, los años de experiencia y la frecuencia de conducción. Por último, se llevaron a cabo diferentes estudios con el objetivo de aportar evidencia concurrente e incremental de la escala.

\section{Método}

\section{Participantes}

Se utilizó una muestra intencional de 378 conductores provenientes de la ciudad de Córdoba, Argentina. La muestra estuvo compuesta por una proporción similar de hombres $(50.3 \%)$ y mujeres $(49.7 \%)$ cuyas edades fluctuaron entre $\operatorname{los} 18$ y 77 años $(M=31.71$; $D E=12.66$ ). El $65 \%$ de la muestra conducía automóvil, el $3.8 \%$ motocicleta, el $4.9 \%$ camioneta (pick up), mientras que el $26.3 \%$ manejaba varios vehículos. Puesto que algunos ítems del DAX no resultan aplicables a motociclistas, se decidió eliminar a estos conductores de forma que la muestra final se conformó por 364 participantes. Con respecto a la frecuencia de conducción, el $64.1 \%$ de los respondientes manejaba diariamente, el $10.8 \%$ entre 4 y 5 días a la semana, el $9.3 \%$ entre dos y tres días a la semana y un porcentaje similar $(9.8 \%)$ al menos una vez a la semana, mientras que el $6.1 \%$ lo hacía de forma esporádica. El tiempo que llevaban conduciendo osciló entre 1 y 61 años $(M=12.04 ; D E=11.84)$. Los participantes fueron contactados en diferentes lugares (universidades $=24.7 \%$; centros de obtención y renovación del permiso de conducir $=75.3 \%$ ) con el propósito de incluir en el estudio una muestra lo suficientemente heterogénea que pudiera reflejar las características existentes en la población de conductores.

\section{Instrumentos}

Driver Anger Expression Inventory (DAX). Se utilizó la versión adaptada al español (HerreroFernández, 2011), compuesta por 53 ítems y un formato de respuesta Likert de 4 puntos (1=Casi nunca; $2=$ Casi siempre) que evalúa cinco modos de expresión de la ira experimentada al volante: Expresión Verbal (e.g., "Insulto a los otros conductores en voz alta"), Expresión Física (e.g., "Le hago gestos con el dedo medio al otro conductor"), Expresión por medio del Vehículo (e.g., "Conduzco pegado al paragolpes del otro conductor"), Expresión Desplazada (e.g., "Grito a la gente que va en el coche conmigo") y Expresión Adaptativa-constructiva (e.g., "Me digo a mí mismo que no merece la pena enojarse"). La consistencia interna obtenida en el estudio español fue adecuada para todos los factores, con coeficientes alfa de Cronbach $(\alpha)$ que oscilaron entre .78 y .91

Driving Anger Scale (DAS). El DAS (Deffenbacher, Oetting \& Lynch (1994) es una escala breve de 14 ítems que recoge el nivel de ira experimentado por los conductores ante diferentes situaciones: avance impedido por otros (e.g., "Alguien está estacionando muy despacio, formando un atasco"); conducción temeraria (e.g., "Alguien está conduciendo en zig-zag); y hostilidad directa (e.g., "Alguien te hace un gesto obsceno por tu forma de conducir"). Cada ítem es respondido mediante una escala tipo Likert de 5 puntos (desde 1=nada; hasta 5=muchísimo), de acuerdo a la intensidad de ira que genera en el conductor. Estudios previos en Argentina (Trógolo et al., 2015) por medio de análisis factoriales exploratorio y confirmatorio ratifican la estructura interna tres factores, como así también la consistencia interna, con valores aceptables para las distintas dimensiones.

\section{Multidimensional Driving Style Inventory} (MDSI). El MDSI (Taubman-Ben-Ari et al., 2004) es un instrumento de autoinforme que evalúa los comportamientos habituales de las 
personas en el contexto de la conducción. Para el presente estudio se utilizó la versión abreviada validada en Argentina por Trógolo y Flores Kanter (2016). La misma cuenta con 16 ítems distribuidos en cuatro factores: conducción riesgosa ( $\alpha=.86$; e.g., "Disfrutar la sensación de manejar al límite"), conducción agresiva $(\alpha=.78$; "Discutir o pelear con otros conductores o con peatones"), conducción prudente ( $\alpha=.72$; e.g., "Intentar conducir con precaución") y conducción disociativa (4 ítems; $\alpha=.69$; e.g., "Por ir distraído o preocupado, darse cuenta tarde de que el vehículo de adelante ha reducido su velocidad, y tener que frenar bruscamente para evitar un choque"). Todos los ítems se responden a través de una escala de Likert que va de 1 (nada) a 6 (mucho) según el grado en que la situación reflejada por el ítem se parece a lo que el respondiente hace o siente cuando conduce.

Cuestionario de datos socio-descriptivos. Se incluyó también un cuestionario ad-hoc con el fin de obtener información socio-demográfica (sexo, edad) y relativa a la conducción (licencia, frecuencia de conducción, tipo de vehículo, etc.)

\section{Procedimiento}

El estudio se llevó a cabo de acuerdo a los lineamientos establecidos para el desarrollo de estudios de carácter instrumental (Carretero-Dios \& Pérez, 2005). De esta forma, se realizó en primer término un análisis cualitativo de los ítems del DAX. A continuación se efectuó un estudio piloto con distintas muestras para examinar el grado de comprensión de los reactivos y obtener una apreciación general de los participantes en relación al instrumento. Posteriormente, se llevó a cabo la recolección de datos con la muestra definitiva mediante la aplicación de una batería compuesta por una hoja con información referida a los objetivos de la investigación y las características de la participación requerida, una hoja con el cuestionario socio-demográfico junto con preguntas relacionadas con la actividad de conducción, y las escalas DAX, DAS y MDSI. La participación en el estudio implicó, en todos los casos, el consentimiento expreso de los participantes, al tiempo que se garantizó el anonimato de las respuestas y la confidencialidad en el manejo de los datos.

\section{Análisis de datos}

Se llevó a cabo un análisis inicial para comprobar la pertinencia de los datos para el análisis factorial y examinar el cumplimientos de los supuestos estadísticos requeridos para el uso de esta técnica (Pérez \& Medrano, 2010). A continuación, se analizó la estructura interna del DAX mediante análisis factorial y se examinó la consistencia interna a través del cómputo del coeficiente alfa de Cronbach. Posteriormente, se llevó a cabo un análisis multivariado de la varianza (MANOVA) con el fin de explorar diferencias en las puntuaciones del DAX en función del sexo, obteniéndose además de las pruebas de significación los tamaños de efecto ( $\eta 2)$, considerando como un tamaño de efecto pequeño valores entre $.01 \mathrm{y} .04$, un tamaño mediano entre .05 y .14 , y grande .14 o más (Cohen, 1988). Para estimar las relaciones entre la edad y las distintas formas de expresión de la ira se calcularon correlaciones parciales controlando el efecto de los años de experiencia, debido al solapamiento empírico entre esta variable y la edad. Por último, se efectuaron diversos análisis bivariados ( $r$ de Pearson y $r_{\mathrm{s}}$ de Spearman) y de regresión múltiple jerárquica con el objetivo de aportar evidencia concurrente e incremental de la escala DAX. Los diferentes tipos de análisis fueron realizados a través de los programas SPSS v20.0 y FACTOR v10.3.

\section{Resultados}

\section{Adaptación lingüística y análisis cualitativo de ítems}

Tal como indica Chahín-Pinzón (2014), aun cuando el idioma sea el mismo, como en el caso del español, las características idiomáticas y culturales pueden variar sensiblemente, siendo aconsejable revisar los ítems para garantizar que el lenguaje sea adecuado en sus aspectos idiomáticos y culturales para la población. Por este motivo, se solicitó a un argentino residente en España desde hace más de tres años que valorara el contenido de los ítems del DAX, en cuanto a los aspectos semánticos y lingüísticos y su potencial aplicabilidad al contexto Argentino. Como resultado, 18 ítems fueron modificados levemente. Posteriormente, se entregó la escala a dos investigadores con conocimientos 
psicométricos y de la variable en estudio, para que examinaran los reactivos considerando los siguientes criterios: (a) pertinencia, (b) relevancia y (c) comprensión. Ambos jueces sugirieron eliminar tres ítems por considerarse que retrataban situaciones improbables o excepcionales, o bien que no eran pertinentes. Los tres ítems corresponden a "Golpeo intencionalmente el paragolpes trasero de otro vehículo", "Me vuelvo loco al volante", y "Le saco la lengua al otro conductor". Dado que también los participantes del estudio piloto refirieron que las situaciones descriptas por estos ítems evocaban situaciones extremas y poco representativas del comportamiento de los conductores, y que el ítem "Me vuelvo loco al volante" generaba dificultades de comprensión, se optó por eliminarlos del análisis posterior.

Asimismo, se identificaron varios pares de ítems con contenidos redundantes. Tal es el caso de los ítems 25 y 46 ("Intento salir del coche y pelearme con el otro conductor"; "Intento salir del coche y agredir al otro conductor"); 7 y 22 ("Encandilo con las luces a otros conductores para molestarles"; "Encandilo al otro conductor en su espejo retrovisor"); 42 y 45 (Digo groserías a los otros conductores en voz alta; "Insulto a los otros conductores en voz alta"); 32 y 55 ("Digo groserías a los otros conductores en voz baja"; "Insulto a los otros conductores en voz baja"). Considerando que un análisis posterior evidenció problemas de multicolinealidad $(r>90)$ y que la presencia de ítems redundantes puede conducir a la aparición de factores adicionales espurios (Lloret-Segura, Ferreres-Traver, HernándezBaeza, \& Tomás-Marco, 2014), se eliminó de cada pareja de ítems redundantes uno de ellos. Como resultado, se descartaron un total de 7 ítems, reteniéndose así 46 ítems de la escala original.

\section{Prueba piloto}

Tras realizar adaptaciones al contenido de los ítems se aplicó el DAX a dos grupos pilotos $(n=12, n=9)$ de forma separada. Por medio de focus group se solicitó a los participantes que brindaran su opinión acerca el instrumento (pertinencia de los ítems, extensión, dificultades de comprensión, tiempo requerido para completar la prueba, etc.). Excepto un ítem ("Me vuelvo loco al volante"), los demás no evidenciaron problemas de comprensión. Todos los participantes indicaron que se trataba de un instrumento útil, interesante, y sencillo de responder. Finalmente, como se indicó, los comportamientos planteados por algunos ítems fueron considerados poco pertinentes, por lo que fueron eliminados.

\section{Análisis de estructura interna}

Se obtuvieron los estadísticos descriptivos de los 46 ítems y se evaluó su capacidad discriminativa. Se encontró que las correlaciones ítem-total corregidas para los ítems 2, 9, 18, 19, $26,33,39,45$ y 51 fueron bajas $(D>.25)$, por lo que fueron retirados. Con los 37 reactivos que discriminaron, se realizó un AF no restricto (Ferrando \& Anguiano-Carrasco, 2010) utilizando matrices de correlaciones policóricas y se apeló al método de mínimos cuadrados no ponderados para la extracción de factores, al comprobarse que varios ítems poseían elevados índices de asimetría (Ferrando \& Lorenzo-Seva, 2014).

La decisión sobre el uso de AF no restricto se basó en el hecho de que aun cuando existe información previa sobre la composición factorial de DAX, la disparidad de los resultados obtenidos en distintas investigaciones impide establecer hipótesis fuertes acerca del número de factores y la cantidad de ítems correspondientes a cada factor, necesario para especificar un modelo factorial. Por este motivo, se optó por un AF no restricto, ya que permite estimar libremente las correlaciones entre los ítems.

Se verificó la adecuación de los datos para factorizar los ítems a través del índice de adecuación muestral de Kaiser-Mayer-Olkin $(K M O=.88)$ y el test de esfericidad de Bartlett, $\chi^{2}(666)=5699.7, \quad p<.001$. Para determinar el número de factores a extraer se utilizó el análisis paralelo de Horn, en la versión implementación óptima propuesta por Timmerman y Lorenzo-Seva (2011). Para la rotación de los factores extraídos se utilizó el método Promin (LorenzoSeva, 1999) al esperarse que los factores estuvieran intercorrelacionados. Los resultados sugieren 5 factores que explican el $59 \%$ de la varianza, GFI=.98, $\mathrm{RMCR}=.048$ (Criterio de Kelley=.052), índice de simplicidad factorial de Bentler $=.98$. La inspección de los residuos 
Tabla 1. Cargas factoriales, estadísticos descriptivos e índices de discriminación para los ítems del DAX

Cargas factoriales

\begin{tabular}{|c|c|c|c|c|c|c|c|c|c|}
\hline Ítems & Factor 1 & Factor 2 & Factor 3 & Factor 4 & Factor 5 & $\mathrm{M}(\mathrm{DE})$ & Asimetria & Curtosis & $D^{(a)}$ \\
\hline item4 & .67 & & & & & $2.69(1.05)$ & -0.11 & -1.22 & .62 \\
\hline item5 & .66 & & & & & $2.54(1.02)$ & -0.01 & -1.12 & .59 \\
\hline item13 & .43 & & & & & $2.79(1.00)$ & -0.32 & -0.99 & .50 \\
\hline item16 & .64 & & & & & $2.54(1.13)$ & 0.04 & -1.39 & .58 \\
\hline item34 & .56 & & & & & $2.55(1.07)$ & -0.03 & -1.25 & .54 \\
\hline item44 & .62 & & & & & $2.52(0.95)$ & 0.07 & -0.91 & .56 \\
\hline item 48 & .72 & & & & & $2.53(1.04)$ & 0.01 & -1.16 & .65 \\
\hline item 17 & & .76 & & & & $1.46(0.88)$ & 1.89 & 2.38 & .47 \\
\hline item20 & & .85 & & & & $1.35(0.74)$ & 2.24 & 4.31 & .60 \\
\hline item23 & & .77 & & & & $1.13(0.44)$ & 3.65 & 13.45 & .47 \\
\hline item 25 & & .69 & & & & $1.10(0.41)$ & 4.46 & 21.42 & .42 \\
\hline item29 & & .91 & & & & $1.32(0.76)$ & 2.54 & 5.51 & .68 \\
\hline item35 & & .50 & & & & $1.67(0.98)$ & 1.29 & 0.54 & .47 \\
\hline item31 & & .41 & & & & $1.30(0.65)$ & 2.39 & 5.54 & .39 \\
\hline item1 & & & .71 & & & $1.54(0.77)$ & 1.45 & 1.58 & .51 \\
\hline item8 & & & .92 & & & $1.26(0.65)$ & 2.75 & 7.12 & .56 \\
\hline item10 & & & .65 & & & $1.34(0.68)$ & 2.10 & 3.84 & .63 \\
\hline item11 & & & .84 & & & $1.22(0.59)$ & 2.99 & 8.78 & .66 \\
\hline item12 & & & .78 & & & $1.46(0.78)$ & 1.75 & 2.38 & .64 \\
\hline item 22 & & & .43 & & & $1.31(0.72)$ & 2.49 & 5.55 & .53 \\
\hline item 28 & & & .56 & & & $1.32(0.71)$ & 2.41 & 5.24 & .61 \\
\hline item36 & & & .64 & & & $1.25(0.62)$ & 2.77 & 7.33 & .45 \\
\hline item46 & & & .52 & & & $1.34(0.68)$ & 2.14 & 4.21 & .61 \\
\hline item41 & & & & .75 & & $1.46(0.80)$ & 1.84 & 2.63 & .53 \\
\hline item 42 & & & & .58 & & $1.56(0.90)$ & 1.52 & 1.20 & .59 \\
\hline item6 & & & & .42 & & $2.01(1.09)$ & 0.66 & -0.93 & .54 \\
\hline item14 & & & & .80 & & $2.83(1.04)$ & -0.33 & -1.14 & .51 \\
\hline item 21 & & & & .59 & & $2.48(1.08)$ & 0.08 & -1.24 & .53 \\
\hline item24 & & & & .38 & & $2.07(1.07)$ & 0.61 & -0.90 & .61 \\
\hline item30 & & & & .50 & & $1.99(1.01)$ & 0.63 & -0.76 & .57 \\
\hline item50 & & -.31 & & .70 & & $2.17(1.07)$ & 0.47 & -1.02 & .44 \\
\hline item47 & & & & & .92 & $1.26(0.64)$ & 2.72 & 7.17 & .67 \\
\hline item49 & & & & & .84 & $1.29(0.66)$ & 2.50 & 5.98 & .61 \\
\hline item37 & & & & & .78 & $1.33(0.67)$ & 2.30 & 5.15 & .63 \\
\hline item53 & & & & & .48 & $1.39(0.74)$ & 2.03 & 3.58 & .47 \\
\hline
\end{tabular}

Nota: Extracción: ULS (mínimos cuadrados no ponderados). Rotación: Promin.

(a) Discriminación del ítem: correlación ítem-total corregida.

estandarizados evidenció que los ítems 3 y 27 poseían residuos elevados (6.21), por lo que fueron eliminados. De esta forma, la escala quedó conformada por 35 ítems distribuidos en 5 factores. Los resultados se sintetizan en la Tabla 1. El Factor 1 posee 7 ítems que explicaron el 32\% de la varianza total y corresponden en su totalidad al factor Expresión Adaptativa-Constructiva. El Factor 2 explicó el $9 \%$ de la varianza total y está compuesto por 7 ítems pertenecientes a la escala de Expresión Física. El Factor 3 explicó el 7\% de la varianza total y está compuesto por 9 ítems que 
Tabla 2. Correlaciones entre edad, variables de conducción y escalas DAX

\begin{tabular}{|c|c|c|c|c|c|}
\hline & $\begin{array}{c}\text { Expresión } \\
\text { adaptativa- } \\
\text { constructiva }\end{array}$ & $\begin{array}{l}\text { Expresión } \\
\text { física }\end{array}$ & $\begin{array}{l}\text { Expresión } \\
\text { mediante el } \\
\text { vehículo }\end{array}$ & $\begin{array}{l}\text { Expresión } \\
\text { desplazada }\end{array}$ & $\begin{array}{l}\text { Expresión } \\
\text { verbal }\end{array}$ \\
\hline 1. Edad & $.20 * *(.13 *)$ & $-.18 * *(-.11 *)$ & $-.28 * *(-.22 * *)$ & $-.14 * *(-.11 *)$ & $-.27 * *(-.17 * *)$ \\
\hline $\begin{array}{l}\text { 2. Experiencia } \\
\text { de conducción } \\
\text { (años) }\end{array}$ & $.13 *(.07)$ & $-.11 *(.06)$ & $-13 *(.-09)$ & $-.05(-.01)$ & $-.20 * *(.07)$ \\
\hline $\begin{array}{l}\text { 3. frecuencia } \\
\text { de conducción }\end{array}$ & .01 & .04 & $.15^{* *}$ & $.12 * *$ & .04 \\
\hline
\end{tabular}

Nota: los coeficientes de correlación parcial se muestran entre paréntesis

${ }^{* *} p<.01$, dos colas. ${ }^{*} p<.05$, dos colas.

pertenecen en su totalidad al factor Expresión Mediante el Vehículo, excepto el ítem 36 ("Intento forzar al otro conductor hacia un lado del camino") que corresponde en su formulación original al factor expresión física. Si bien posee cargas en un factor diferente del esperado, se mantuvo ya que su contenido es coherente con el factor. El Factor 4 explicó el 6\% de la varianza total y abarca 8 ítems que pertenecen en su totalidad al factor Expresión Verbal. Por último, el Factor 5 explicó el 5\% de la varianza total y está compuesto por 4 ítems que pertenecen al factor Expresión Desplazada. Todos los ítems mostraron cargas factoriales superiores a $.30 \mathrm{y}$ adecuada capacidad de discriminación. Finalmente, los análisis de consistencia interna fueron satisfactorios, con valores superiores a .70 en todos los factores.

\section{Diferencias según sexo, edad, años de experiencia y frecuencia de conducción}

Los resultados del MANOVA indicaron diferencias significativas por sexo, Wilks $\lambda=.96$, $F(5,359)=3.26, p=.007, \eta_{\mathrm{p}}{ }^{2}=.043$. Específicamente, el ANOVA univariado mostró diferencias en la subescala expresión mediante el vehículo, $F(1$, 374) $=14.05, p<.001, \eta_{\mathrm{p}}{ }^{2}=.039$, siendo los hombres quienes puntuaron más alto. No se observaron diferencias significativas para el resto de las dimensiones del DAX, expresión física $F(1$, 374)=2.89, $p=.09$; expresión adaptativaconstructiva, $F(1,374)=1.30, p=.25$; expresión verbal, $\quad F(1, \quad 374)=0.42, \quad p=.51 ; \quad$ expresión desplazada, $F(1,374)=2.04, p=.15$.

Con respecto a la edad, las correlaciones parciales con las diferentes formas de expresión (controlando el efecto de los años de experiencia) indicaron correlaciones positivas con expresión adaptativa-constructiva y correlaciones negativas con las distintas formas de expresión agresiva directa Por otra parte, cuando se controló el efecto de la edad, las correlaciones parciales entre los años de experiencia y las distintas formas de expresión no fueron significativas. Por último, la frecuencia de manejo correlacionó de manera positiva con la expresión de ira mediante el vehículo y la expresión desplazada (Tabla 2).

\section{Validez concurrente}

Se evaluó la validez concurrente del DAX analizando las correlaciones con la escala de ira en la conducción (DAS) y el inventario multidimensional de estilos de conducción (MDSI). Debido a la distribución asimétrica (índices superiores a \pm 2.00 ) que presentaron los puntajes correspondientes a las dimensiones expresión física, expresión mediante el vehículo y expresión desplazada, se computó el coeficiente de correlación por rangos de Spearman $\left(r_{\mathrm{s}}\right)$. Para el resto de los factores, se obtuvo el coeficiente de correlación de Pearson ( $r$ ). En todos los casos, se obtuvieron correlaciones significativas y en la dirección esperada entre los distintos factores del DAX y las dimensiones del DAS y del MDSI, obteniéndose de esta manera evidencia concurrente de la escala. Una descripción más detallada de los resultados se ofrece en la Tabla 3.

\section{Validez incremental}

Se efectuaron una serie análisis de regresión múltiple jerárquica para comprobar si las dimensiones del DAX contribuían a explicar los comportamientos de conducción una vez controlado el efecto de otras variables explicativas relevantes como el sexo y la edad, y la ira en la conducción. Para ello se conformaron cuatro 
Tabla 3. Correlaciones entre los factores del DAX y medidas de ira (DAS) y comportamientos en la conducción (MSDI)

\begin{tabular}{|c|c|c|c|c|c|c|c|c|c|c|c|c|c|c|}
\hline & $\mathrm{M}$ & $\mathrm{DE}$ & 1 & 2 & 3 & 4 & 5 & 6 & 7 & 8 & 9 & 10 & 11 & 12 \\
\hline $\begin{array}{l}1 . \\
\text { Expresión adaptativa } \\
(\mathrm{DAX})^{\mathrm{a}}\end{array}$ & 2.59 & 0.73 & - & & & & & & & & & & & \\
\hline $\begin{array}{l}\text { 2. Expresión física } \\
(\mathrm{DAX})^{\mathrm{b}}\end{array}$ & 1.33 & 0.46 & $-.37^{* *}$ & - & & & & & & & & & & \\
\hline $\begin{array}{l}\text { 3. Expresión } \\
\text { mediante el vehículo } \\
(\mathrm{DAX})^{\mathrm{b}}\end{array}$ & 1.37 & 0.47 & $-.40^{* *}$ & $.58^{* *}$ & - & & & & & & & & & \\
\hline $\begin{array}{l}\text { 4. Expresión } \\
\text { desplazada }(\mathrm{DAX})^{\mathrm{b}}\end{array}$ & 1.31 & 0.53 & $-.24^{* *}$ & $.34^{* *}$ & $.33^{* *}$ & - & & & & & & & & \\
\hline $\begin{array}{l}\text { 5. Expresión verbal } \\
(\mathrm{DAX})^{\mathrm{a}}\end{array}$ & 1.84 & 0.60 & $-.37^{* *}$ & $.60^{* *}$ & $.48^{* * *}$ & $.41^{* * *}$ & - & & & & & & & \\
\hline $\begin{array}{l}\text { 6. Avance impedido } \\
\text { por otros (DAS) }\end{array}$ & 14.82 & 4.38 & $-.29^{* *}$ & $.31^{* *}$ & $.35^{* *}$ & $.33^{* *}$ & $.47^{* *}$ & - & & & & & & \\
\hline $\begin{array}{l}\text { 7. Hostilidad directa } \\
\text { (DAS) }\end{array}$ & 5.46 & 2.61 & $-.25^{* *}$ & $.18^{* *}$ & $.16^{* *}$ & $.23^{* *}$ & $.29^{* *}$ & $.43^{* * *}$ & - & & & & & \\
\hline $\begin{array}{l}\text { 9. Conducción } \\
\text { temeraria (DAS) }\end{array}$ & 16.27 & 3.84 & $-.14^{* * *}$ & $.17^{* *}$ & $.21^{* * *}$ & $.27^{* *}$ & $.29^{* * *}$ & $.53^{* *}$ & $.38^{* *}$ & - & & & & \\
\hline $\begin{array}{l}\text { 10. Conducción } \\
\text { riesgosa (MDSI) }\end{array}$ & 9.59 & 5.12 & $-.22 * *$ & $.33^{* *}$ & $.49^{* *}$ & $.19^{* * *}$ & $.33^{* *}$ & $.34^{* * *}$ & $.19^{* *}$ & $.15^{* *}$ & - & & & \\
\hline $\begin{array}{l}\text { 11. Conducción } \\
\text { agresiva (MDSI) }\end{array}$ & 10.44 & 4.45 & $-.45^{* *}$ & $.58^{* * *}$ & $.57^{* *}$ & $.39^{* *}$ & $.67^{* * *}$ & $.54^{* * *}$ & $.32^{* *}$ & $.35^{* *}$ & $.54^{* *}$ & - & & \\
\hline $\begin{array}{l}\text { 12. Conducción } \\
\text { prudente (MDSI) }\end{array}$ & 18.98 & 3.70 & $.36^{* *}$ & $-.14^{* *}$ & $-.24^{* *}$ & $.17^{-}$ & $-.11^{*}$ & $.17^{-}$ & $-.12^{*}$ & .01 & $.20^{-}$ & $-.22^{* * *}$ & - & \\
\hline $\begin{array}{l}\text { 13. Conducción } \\
\text { disociativa (MDSI) }\end{array}$ & 8.38 & 3.34 & $-.13 *$ & $.25^{* *}$ & $.26^{* *}$ & $.21^{* *}$ & $.19^{* *}$ & $.31^{* * *}$ & $.21^{* *}$ & $.22^{* *}$ & $.22^{* *}$ & $.34^{* *}$ & $.18^{-}$ & - \\
\hline
\end{tabular}

Nota: ${ }^{\text {a }}$ Correlación de Pearson. ${ }^{\mathrm{b}}$ Correlación por rangos de Spearman ${ }^{* *} p<.01$, dos colas. ${ }^{*} p<.05$, dos colas.

modelos de regresión tomando como variables dependientes en cada modelo los diferentes estilos de conducción. Como variables predictoras se introdujeron, en un primer paso, el sexo y la edad. En un segundo paso se introdujeron las dimensiones del DAS. Por último, se introdujeron las dimensiones del DAX. Los resultados obtenidos mostraron que las puntuaciones del DAX mejoran significativamente la capacidad explicativa en todos los modelos, incrementando la varianza explicada entre un $5 \%$ y un $27 \%$, verificándose así la contribución incremental de la escala (ver Tabla 4).

\section{Discusión}

En el presente trabajo se han estudiado las propiedades psicométricas del DAX en conductores de la población general de la ciudad de Córdoba, Argentina. Los resultados obtenidos son semejantes a los encontrados en la versión original del instrumento (Deffenbacher et al.,
2002). Sin embargo, y en consonancia con la adaptación realizada en España (HerreroFernández, 2011), el factor de expresión desplazada se mantuvo, al evidenciar una adecuada consistencia interna. De esta manera, se identificaron a través del DAX cinco formas de expresión de la ira en conductores argentinos: expresión adaptativa-constructiva, expresión verbal, expresión física, expresión mediante el vehículo, y expresión desplazada. Al igual que en otras investigaciones (Herrero-Fernández, 2012; Sullman et al., 2013, 2015; Villeux \& Delhomme, 2010), la expresión adaptativa-constructiva y la expresión verbal de la ira fueron las formas de expresión más comunes en los conductores. En cambio, la expresión desplazada fue la menos frecuente.

Es conveniente señalar que durante el proceso de validación se eliminaron un total de 18 ítems, muchos de ellos bien por su contenido redundante, o bien por las bajas correlaciones ítem-total que mostraron con sus respectivos factores. A pesar de 
Tabla 4. Validez incremental del DAX

\begin{tabular}{|c|c|c|c|c|c|c|c|c|}
\hline \multirow[b]{2}{*}{ Predictor } & \multicolumn{2}{|c|}{ Conducción riesgosa } & \multicolumn{2}{|c|}{$\begin{array}{c}\text { Conducción } \\
\text { agresiva }\end{array}$} & \multicolumn{2}{|c|}{$\begin{array}{l}\text { Conducción } \\
\text { prudente }\end{array}$} & \multicolumn{2}{|c|}{$\begin{array}{c}\text { Conducción } \\
\text { disociativa }\end{array}$} \\
\hline & $\Delta R^{2}$ & $\beta$ & $\Delta R^{2}$ & $\beta$ & $\Delta R^{2}$ & $\beta$ & $\Delta R^{2}$ & $\beta$ \\
\hline Paso 1 & $.126^{* * *}$ & & $.058 * * *$ & & .012 & & .005 & \\
\hline Sexo & & $-.28 * * *$ & & $-.15 * *$ & & .03 & & -.01 \\
\hline Edad & & -.23 & & $-.19 * * *$ & & .11 & & .07 \\
\hline Paso 2 & $.102 * * *$ & & $.284 * * *$ & & .014 & & $.087 * * *$ & \\
\hline $\begin{array}{l}\text { Avance impedido por } \\
\text { otros }\end{array}$ & & $.28 * * *$ & & $.44 * * *$ & & -.12 & & $.23^{* * *}$ \\
\hline Hostilidad directa & & .08 & & .06 & & -.05 & & .05 \\
\hline Conducción temeraria & & -.01 & & .10 & & .08 & & .06 \\
\hline Paso 3 & $.114 * * *$ & & $.270 * * *$ & & $.136^{* * *}$ & & $.049 * *$ & \\
\hline Expresión física & & .03 & & $.16^{* *}$ & & .00 & & $.14 *$ \\
\hline $\begin{array}{l}\text { Expresión mediante el } \\
\text { vehículo }\end{array}$ & & $.35 * * *$ & & $.17 * * *$ & & $-.13 * *$ & & $.14^{*}$ \\
\hline Expresión desplazada & & -.06 & & .05 & & $-.14 * *$ & & .05 \\
\hline Expresión verbal & & .06 & & $.31 * * *$ & & $-.20 * *$ & & -.06 \\
\hline $\begin{array}{l}\text { Expresión adaptativa- } \\
\text { Constructiva }\end{array}$ & & .03 & & $-.11 * *$ & & $.33 * * *$ & & .00 \\
\hline
\end{tabular}

Nota: sexo: variable ficticia $(1=$ hombre; $2=$ mujer $)$

$* * * p<.001 ; * *$

$p<.01 ; * p<.05$

la reducción significativa en el número de ítems respecto del instrumento original, la escala conserva la estructura factorial original y los coeficientes de consistencia interna muestran valores aceptables y óptimos para los diferentes factores.

Los resultados obtenidos en cuanto a la edad son consistentes con los estudios previos al señalar que los conductores jóvenes obtienen puntuaciones más altas en las distintas formas de expresión agresiva de la ira (verbal, física, mediante el vehículo y desplazada) mientras que los conductores de mayor edad lo hacen en expresión adaptativa-constructiva (Deffenbacher et al., 2007; Jovanović et al., 2011; Sârbescu, 2012; Stephens \& Sullman, 2014; Villeux \& Delhomme, 2010). Estos resultados se mantienen tras controlar el efecto de la experiencia de conducción. Por el contrario, las correlaciones obtenidas entre la experiencia de conducción y las diferentes formas de expresión de la ira no fueron significativas una vez controlado el efecto de la edad. Estos resultados sugieren que las relaciones reportadas previamente (Jovanović et al., 2011; 2011; Stephens \& Sullman, 2014;
Sullman et al., 2013, 2015) entre los años de experiencia y las formas de expresión son espurias, y que las diferencias encontradas se deben a la edad de los conductores.

Con respecto al sexo, los resultados evidenciaron diferencias en la expresión mediante el vehículo, siendo los hombres quienes más tienden a expresar la ira de esta forma, aunque el tamaño del efecto fue bajo (Cohen, 1988), en coherencia con diferentes estudios (Stephens \& Sullman, 2014; Sullman, 2015). Sin embargo, contrasta con otras investigaciones en las que no se ha encontrado ninguna diferencia debida al sexo (Deffenbacher et al., 2007; Ge et al., 2015; Herrero-Fernández, 2011; Villeux \& Delhomme, 2010). Presumiblemente, la ausencia de diferencias podría deberse a las características particulares de la conducción (anonimato), que ofrecería una oportunidad única para la expresión de la ira, al pasar desapercibida por el otro conductor o por la menor probabilidad que desencadene una agresión directa por parte de quien la recibe, lo que parecería darse indistintamente en hombres y mujeres (EllisonPotter, Bell \& Deffenbacher, 2001). De cualquier 
manera, atendiendo a las discrepancias existentes, son necesarios más estudios que permitan aclarar estos hallazgos inconsistentes sobre las diferencias de sexo.

El análisis de la frecuencia de conducción mostró que los conductores que manejaban asiduamente expresan su ira mediante el vehículo y de manera desplazada con mayor frecuencia, aunque las relaciones obtenidas fueron débiles en todos los casos. Estos resultados son consistentes con los observados en estudios anteriores ( $\mathrm{Ge}$ et al., 2015; Sullman, 2015; Sullman et al., 2013, 2015) y sugieren que las personas que conducen con mayor frecuencia presentan una mayor vulnerabilidad a expresar agresivamente su enfado, presumiblemente debido a una mayor exposición a eventos frustrantes en el contexto del tránsito. No obstante, creemos que estos resultados deben ser interpretados con cautela y, en vista de las discrepancias existentes en la literatura, sería recomendable realizar nuevos estudios que incorporen variables predisposicionales como la expresión de ira-rasgo (Leibovich de Figueroa, Schmidt \& Gol, 2001) y analicen los efectos interactivos con la frecuencia de manejo en la expresión de la ira en la conducción.

Por otra parte, el patrón de correlaciones observadas entre las escalas del DAX y las medidas de ira en la conducción y comportamiento en la conducción fue, en todos los casos, significativo y coherente en términos teóricos, aportando así evidencia complementaria de validez concurrente. En concreto, tal como se esperaba en base a los resultados de diferentes investigaciones (Deffenbacher et al., 2001, 2002, 2004, Esiyok et al., 2007; Ge et al., 2015; Herrero-Fernández, 2011; Sullman, 2015), los conductores con mayores puntajes en las diferentes formas de expresión agresiva evidenciaron también mayores niveles de ira en la conducción, mayor frecuencia de comportamientos desadaptativos (i.e., conducción arriesgada, agresiva y disociativa) y menores comportamientos prudentes en la conducción, al tiempo que quienes obtuvieron puntajes más altos en expresión adaptativa-constructiva evidenciaron el patrón de relaciones opuesto. Finalmente, los diferentes análisis de regresión múltiple jerárquica mostraron la capacidad de la escala DAX para explicar de manera significativa distintos comportamientos de conducción, por encima de otras variables explicativas relevantes como la edad, el sexo y la ira en la conducción, lo que constituye una evidencia de validez incremental de la escala.

En síntesis, los resultados obtenidos son globalmente satisfactorios y respaldan la utilización del DAX en la población Argentina. No obstante, se requieren estudios adicionales que evalúen otras cualidades psicométricas importantes como la estabilidad temporal del instrumento (confiabilidad test-retest), la validez convergente y discriminante y su robustez frente a sesgos de respuesta. Asimismo, sería deseable llevar a cabo nuevas investigaciones mediante análisis factorial confirmatorio que evalúen la adecuación de la estructura factorial obtenida.

Los aspectos emocionales de los conductores han recibido una atención progresiva en los últimos años (Jeon et al., 2014; Taubman-BenAri, 2012), particularmente el estudio de la agresividad en la conducción, la cual puede considerarse un problema en crecimiento (Ledesma, Peltzer, \& Poó, 2008). Sin embargo, las investigaciones que se han ocupado del tema provienen de otras latitudes, existiendo una carencia de estudios en el contexto local. El presente trabajo constituye un aporte en este sentido que permitirá impulsar líneas de investigación en torno a la expresión de la ira en conductores. Además, permite disponer de un instrumento útil para identificar conductores con tendencia a expresar agresivamente la ira y determinar el impacto de intervenciones psicológicas que tengan por objetivo la reeducación emocional de conductores.

\section{Referencias}

Alonso, F., Esteban, C., Calatayud, C., Alamar, B., \& Egido, A. (2006). Emociones y conducción: Teoría y fundamentos. España: Colección Cuadernos de Reflexión Attitudes

Casullo, M. (2009). La evaluación psicológica: Modelos, técnicas y contextos. Revista Iberoamericana de Diagnóstico y Evaluación - e Avaliação Psicológica, 27, 9-28.

Chahín-Pinzón, N. (2014). Aspectos a tener en cuenta cuando se realiza una adaptación de 
test entre diferentes culturas. Psychologia: Avances de la Disciplina, 8, 109-112.

Cohen, J. (1988). Statistical power analysis for the behavioral sciences (2nd ed.). Hillsdale, NJ: Erlbaum.

Dahlen, E., \& Ragan, K. (2004). Validation of the propensity for Angry Driving Scale. Journal of Safety Research, 35, 557-563. doi:10.1016/j.jsr.2004.09.002

Deffenbacher, J., Kemper, C., \& Richards, T. (2007). The Driving Anger Expression Inventory: A validity study with community college student drivers. Journal of Psychopathology and Behavioral Assessment, 29, 220-230. doi: 10.1007/s10862-007-9049-x

Deffenbacher, J., Lynch, R., Deffenbacher, D., \& Oetting, E. (2001). Further evidence of reliability and validity for the Driving Anger Expression Inventory. Psychological Reports, 89, 535-540. doi: 10.2466/pr0.2001.89.3.535

Deffenbacher, J., Lynch, R., Filetti, L., Dahlen, E., \& Oetting, E. (2003). Anger, aggression, risky behavior, and crash-related outcomes in three groups of drivers. Behaviour Research and Therapy, 41, 333-349. doi:10.1016/S0005-7967(02)00014-1

Deffenbacher, J., Lynch, R., Oetting, E., \& Swaim, R. (2002). The Driving Anger Expression Inventory: A measure of how people express their anger on the road. Behaviour Research and Therapy, 40, 717737. doi:10.1016/S0005-7967(01)00063-8

Deffenbacher, J., Lynch, R., Oetting, E., \& Yingling, D. (2001). Driving anger: Correlates and a test of state-trait theory. Personality and Individual Differences, 31, 1321-1331. doi:10.1016/S0191-8869(00)00226-9

Deffenbacher, J., White, G., \& Lynch, R. (2004). Evaluation of two new scales assessing driving anger: The Driving Anger Expression Inventory and the Driver's Angry Thoughts Questionnaire. Journal of Psychopathology and Behavioral Assessment, 26, 87-99. doi: 10.1023/B:JOBA.0000013656.68429.69

Ellison-Potter, P., Bell, P., \& Deffenbacher, J. (2001). The effects of trait driving anger, anonymity, and aggressive stimuli on aggressive driving behavior. Journal of Applied Social Psychology, 31, 431-443. doi: 10.1111/j.1559-1816.2001.tb00204.x
Escalante, M., Gómez, R., Cuasnicu, A., \& Ansaldo, S. (2013). El uso indebido de drogas y la consulta de emergencia. Quinto estudio nacional. Argentina: Secretaría de Programación para la Prevención de la Drogadicción y Lucha contra el Narcotráfico. Recuperado de http://www.observatorio.gob. ar/media/k2/attachments/VZEstudioZNaciona IZdelZUsoZIndebidoZdeZDrogasZyZlaZCons ultaZdeZEmergencia.ZAoZ2013.pdf

Escanés, G., Agudelo-Botero, M., \& Cardona, D. (Agosto, 2014). El impacto de las muertes por atropellos y colisiones de tránsito en la esperanza de vida en Argentina, Chile, Colombia y México, 2000-2011. Trabajo presentado en el VI Congreso de la Asociación Latinoamericana de Población, Lima, Perú.

Esiyok, B., Yasak, Y., \& Korkusuz, I. (2007). Anger expression on the road: Validity and reliability of the Driving Anger Expression Inventory. Turkish Journal of Psychiatry, 18, 231-243.

Evans, L. (1996). The dominate role of driver behavior in traffic safety. American Journal of Public Health, 86, 784-786.

Ferrando, P., \& Anguiano-Carrasco, C. (2010). El análisis factorial como técnica de investigación en psicología. Papeles del psicólogo, 31, 18-33.

Ferrando, P., \& Lorenzo-Seva, U. (2014). El análisis factorial exploratorio de los ítems: algunas consideraciones adicionales. Anales de psicología, 30, 1170-1175. http://dx.doi.or g/10.6018/analesps.30.3.199991

Fleitas, D. (2010). Accidentes de tránsito en argentina. Segundo informe / 1997- 2008. Recuperado de http://www.portalseguridad.or g/attachments/Accidentes_de_Transito_en_Ar gentina_2010_final.pdf

Furr, M. (2011). Scale construction and psychometrics for social and personality psychology. London: Sage

Ge, Y., Qu, W., Zhang, Q., Zhao, W., \& Zhang, K. (2015). Psychometric adaptation of the Driving Anger Expression Inventory in a Chinese sample. Transportation Research Part F: Traffic Psychology and Behaviour, 33, 75-86. doi: 10.1016/j.trf.2015.07.008 
Gras, M., Font-Mayolas, S., Patiño, J., Baltasar, A., Planes, M., \& Sullman, M. (2015). Resilience and the expression of driving anger. Transportation Research Part F: Traffic Psychology and Behaviour. doi: 10.1016/j.trf.2015.09.005

Herrero-Fernández， D. (2011). Psychometric adaptation of the Driving Anger Expression Inventory in a Spanish sample: Differences by age and gender. Transportation Research Part F: Traffic Psychology and Behaviour, 14, 324-329. doi: 10.1016/j.trf.2011.03.001

Herrero-Fernández, D. (2012). Análisis de diferencias en la magnitud de la ira provocada por distintos eventos del tráfico y en las formas de expresarla. Securitas Vialis, 13, 1123.

Jeon, M., Walker, B., \& Yim, J. (2014). Effects of specific emotions on subjective judgment, driving performance, and perceived workload. Transportation Research Part F: Traffic Psychology and Behaviour, 24, 197-209. doi: 10.1016/j.trf.2014.04.003

Jovanović, D., Lipovac, K., Stanojević, P., \& Stanojević, D. (2011). The effects of personality traits on driving-related anger and aggressive behaviour in traffic among Serbian drivers. Transportation Research Part F: Traffic Psychology and Behaviour, 14, 43-53. doi:10.1016/j.trf.2010.09.005

Ledesma, R., Peltzer, R., \& Poó, F. (2008). Análisis de la producción en Psicología del Tránsito mediante PsycINFO (2000-2006). Psic: Revista de Psicología da Vetor Editora, 9, 11-24.

Leibovich de Figueroa, N., Schmidt, V., \& Gol, S. (2001). El Inventario de Expresión de Enojo Estado-Rasgo (STAXI) y su uso en diferentes poblaciones. Revista Iberoamericana de Diagnóstico y Evaluación - e Avaliação Psicológica, 11, 55-74.

Leveau, C., \& Úbeda, C. (2012). Muertes por lesiones de tránsito en Argentina: Un análisis espacial para el período 2001-2009. Revista Panamericana de Salud Pública, 31, 439-442.

Li, F., Yao, X., Jiang, L., \& Li, Y. (2014). Driving anger in China: Psychometric properties of the Driving Anger Scale (DAS) and its relationship with aggressive driving.
Personality and Individual Differences, 68, 130-135. doi:10.1016/j.paid.2014.04.018

Lloret-Segura, S., Ferreres-Traver, A., HernándezBaeza, A., \& Tomás-Marco, I. (2014). El análisis factorial exploratorio de los ítems: Una guía práctica, revisada y actualizada. Anales de Psicología, 30, 1151-1169. doi:10.6018/analesps.30.3.199361

Lorenzo-Seva, U. (1999). Promin: A method for oblique factor rotation. Multivariate Behavioral Research, 34, 347-356. doi:10.1207/S15327906MBR3403_3

Maxwell, J., Grant, S., \& Lipkin, S. (2005). Further validation of the Propensity for Angry Driving Scale in British drivers. Personality and Individual Differences, 38, 213-224. doi:10.1016/j.paid.2004.04.002

Ministerio de Salud de la Nación Argentina (2013). Descripción epidemiológica de la mortalidad por lesiones de causas externas en Argentina. Boletín de Vigilancia de Enfermedades no transmisibles y factores de Riesgo (Núm. 6). Recuperado de http://www.msal.gob.ar/ent/images/stories/vig ilancia/pdf/2014-03_boletin-epideomologia06.pdf

Moheb, N., \& Ram, U. (2010). Cross-cultural study of stress and anger. Procedia - Social and Behavioral Sciences, 5, 1765-1769. doi:10.1016/j.sbspro.2010.07.361

OMS (2013). Global status report on road safety. Geneva: World Helath Organization. Recuperado de: http://apps.who.int/iris/bitstre am/10665/44122/1/9789241563840_eng.pdf

Pérez, E., \& Medrano, L. A. (2010). Análisis factorial exploratorio: Bases conceptuales y metodológicas. Revista Argentina de Ciencias del Comportamiento (RACC), 2, 58-66.

Petit, L. (2014). El factor humano en el sistema tránsito y seguridad vial y el modelo interaccional comportamental de tránsito. Psiencia. Revista Latinoamericana de Ciencia Psicológica, 6, 48-54. doi: 10.5872/psiencia/6 .1 .121

Poó, F. (2014). Evaluación multidimensional del estilo de conducción. Su relación con variables psicológicas y comportamientos de riesgo (Tesis doctoral inédita). Universidad Nacional de Mar del Plata, Mar del Plata, Argentina. 
Poó, F., Taubman-Ben-Ari, O., Ledesma, R., \& Díaz-Lázaro, C. (2013). Reliability and validity of a spanish-language version of the multidimensional driving style inventory. Transportation Research Part F: Traffic Psychology and Behaviour, 17, 75-87. doi: http://dx.doi.org/10.1016/j.trf.2012.10.003

Sârbescu, P. (2012). Aggressive driving in Romania: Psychometric properties of the Driving Anger Expression Inventory. Transportation Research Part F: Traffic Psychology and Behaviour, 15, 556564. doi: 10.1016/j.trf.2012.05.009

Sârbescu, P., Stanojević, P., \& Jovanović, D. (2014). A cross-cultural analysis of aggressive driving: Evidence from Serbia and Romania. Transportation Research Part F: Traffic Psychology and Behaviour, 24, 210217. doi:10.1016/j.trf.2014.04.002

Stephens, A., \& Sullman, M. J. (2014). Development of a short form of The Driving Anger Expression Inventory. Accident Analysis and Prevention, 72, 169-176. doi: 10.1016/j.aap.2014.06.021

Sullman, M. (2015). The expression of anger on the road. Safety Science, 72, 153-159. doi: 10.1016/j.ssci.2014.08.013

Sullman, M., Stephens, A., \& Yong, M. (2014). Driving anger in Malaysia. Accident Analysis and Prevention, 71, 1-9. doi:10.1016/j.aap.2014.04.019

Sullman, M., Stephens, A., \& Yong, M. (2015). Anger, aggression and road rage behavior in Malaysian drivers. Transportation Research Part F: Traffic Psychology and Behavior, 29, 70-82. doi: 10.1016/j.trf.2015.01.006

Tasca, L. (2000). A review of the literature on aggressive driving research. Aggressive Driving Issues Conference, Sponsored by the Ministry of Transportation of Ontario, Canada, in Cooperation with the Transportation Research Board. Recuperado de http://www.aggressive.drivers.com/papers/ tasca/tasca.pdf.

Taubman-Ben-Ari, O. (2012). The effects of positive emotion priming on self-reported reckless driving. Accident Analysis \& Prevention, 45, 718- 725. doi:10.1016/j.aap.2 011.09.039
Taubman-Ben-Ari, O., Mikulincer, M., \& Gillath, O. (2004). The multidimensional driving style inventory-scale construct and validation. Accident Analysis and Prevention, 36, 323332. doi:10.1016/S0001-4575(03)00010-1

Taubman-Ben-Ari, O., Mikulincer, M., \& Gillath, O. (2004). The multidimensional driving style inventory-scale construct and validation. Accident Analysis and Prevention, 36, 323332. doi:10.1016/S0001-4575(03)00010-1

Timmerman, M. \& Lorenzo-Seva, U. (2011). Dimensionality Assessment of Ordered Polytomous Items with Parallel Analysis. Psychological Methods, 16, 209-220. http://dx.doi.org/10.1037/a0023353

Trógolo, M., \& Flores Kanter, P. E. (Octubre, 2016). Nuevos estudios de validación del inventario multidimensional de estilos de conducción. Trabajo presentado en el II Congreso Latinoamericano de la Asociación para el Avance de la Ciencia Psicológica, Buenos Aires, Argentina.

Trógolo, M., Flores Kanter, P. E., Pareja, A., \& Medrano, L. A. (2015). Estudios de validación de la escala abreviada de ira en la conducción (DAS) en conductores argentinos. Manuscrito enviado para publicación.

Villieux, A., \& Delhomme, P. (2010). Driving anger and its expressions: Further evidence of validity and reliability for the Driving Anger Expression Inventory French adaptation. Journal of Safety Research,41, 417-422. doi: 10.1016/j.jsr.2010.08.003

Wiesenthal, D., Hennessy, D., \& Gibson, P. (2000). The Driving Vengeance Questionnaire (DVQ): The development of a scale to measure deviant drivers' attitudes. Violence and Victims, 15, 115-136. 